\title{
Optimal Capacitor Placement Techniques in Transmission and Distribution Networks to Reduce Line Losses and Voltage Stability Enhancement: A Review
}

\author{
Om Prakash Mahela ${ }^{1}$, Devendra Mittal $^{2}$ Lalit Goyal ${ }^{3}$ \\ 1, 3 (Junior Engineer, Rajasthan Rajya Vidhyut Prasaran Nigam Ltd., Jaipur, India) \\ ${ }^{2}$ (Assistant Professor, Department of Electrical Engineering, Jagannath University Jaipur, India)
}

\begin{abstract}
The various optimal capacitor placement techniques on transmission and distributions lines for line losses reduction and enhancement of voltage stability in the power system network have been proposed so far in different papers. Optimal Capacitor placement is an optimization problem which has an objective to define the sizes and locations of capacitors to be installed. This paper focuses on the optimal capacitor placement and sizing problem formulation and analytical as well as heuristic artificial intelligence optimization methods for optimal capacitor placement and sizing. This paper helps the researchers to know about the different methods presented so far for optimal capacitor placement and sizing in transmission and distribution networks so that further work on optimal capacitor placement can be carried out for better results.
\end{abstract}

Keywords - capacitor sizing, distribution system, heuristic techniques, optimal capacitor placement, reactive power, transmission system, voltage stability.

\section{Introduction}

Electric transmission system is the intermediate stage in the transfer of electrical energy from the central generating station to the consumers and distribution system is the last stage in delivery of power to the consumer by the utility. With the policy of installation of large pit head thermal power plants and nuclear power plants the electric transmission and distribution networks are becoming complex. The flow of reactive power in an electric network produces supplementary power loss and increases the voltage drop [1]. The losses in the transmission network of Rajasthan state of India was 4.86\% in the year 2011-12 [2] and researches show that about $13 \%$ of the power produced in the system wastes as ohmic losses [3]. In India, collective of all states, in 2008 the technical and non technical losses are accounted as $23 \%$ of the total input energy [4].

The shunt capacitors are effective tool in power and energy loss reduction. These are commonly used in power system network for reactive power compensation. They reduce the transmission \& distribution losses and improve the overall power factor of the system whose effect is seen in terms of higher returns in revenue metering. An indirect advantage of capacitor placement is seen as a reduction in the amount of MVAR transportation required at higher voltage levels on transmission system. They also help in maintaining the voltage profile within acceptable limits. The amounts of benefit that can be achieved by placing the capacitors depend on how much and how the capacitors are placed in the distribution and transmission networks. The problem of locating and sizing shunt capacitors in the power system has been a challenge for power system planners and researchers. In this work, an analysis of analytical optimization as well as heuristic optimization techniques that solve the optimal capacitor placement and sizing in electrical power networks proposed by the researchers recently are presented

\section{Optimal Capacitor Placement and Sizing Problem Formulation}

The objective of capacitor placement in the electric network is to minimize the losses and improve voltage profile. The load and capacitor model, objective function, constraints and power loss calculations are described in this section.

\subsection{Load and capacitor model}

The loads and capacitors are modeled as impedance. The impedance model of loads and capacitors are given by Eq. (1) and Eq. (2):

$$
\begin{aligned}
& Z_{\text {Load }_{i}}=R_{\text {Load }_{i}}+j X_{\text {load }_{i}} \\
& \mathrm{i}=1,2,3, \ldots \ldots \ldots \ldots, \mathrm{NL} \\
& \text { Where } \quad \mathrm{NL}=\text { number of loads } \\
& Z_{\text {Load }_{i}}=\text { load impedance of } \mathrm{i}^{\text {th }} \text { load } \\
& R_{\text {Load }_{i}}=\text { load resistance of } \mathrm{i}^{\text {th }} \text { load }
\end{aligned}
$$




$$
\begin{array}{r}
X_{\text {load }_{i}}=\text { load reactance of } \mathrm{i}^{\text {th }} \text { load } \\
Z_{C_{k}}=-j X_{C_{k}}
\end{array}
$$

$\mathrm{k}=1,2,3, \ldots$ NC

Where $\quad \mathrm{NC}=$ number of capacitors

$Z_{C_{k}}=$ impedance of $\mathrm{k}^{\text {th }}$ capacitor

$X_{C_{k}}=$ reactance of $\mathrm{k}^{\text {th }}$ capacitor

\subsection{Objective function}

The three-phase system is considered as balanced and loads are assumed as time invariant. Mathematically, the objective function of the problem is minimizing the loss and voltage deviation. This function is:

$$
F=W_{1} \times P_{\text {loss }}+W_{2} \times \sum_{i=1}^{n}\left(1-v_{i}\right)^{2}
$$

Where $\mathrm{W}_{1}$ and $\mathrm{W}_{2}$ are objective function coefficient for power loss and objective function coefficient for voltage deviation. $P_{\text {loss }}$ is total loss in transmission system. $v_{i}$ is voltage magnitude of $\mathrm{i}^{\text {th }}$ load

\subsection{Constraints}

The voltage magnitude at each bus must be maintained within its limits and is expressed as:

$$
v_{\min }<\left|v_{i}\right|<v_{\max }
$$

Where $\left|v_{i}\right|$ is voltage magnitude of $\mathrm{i}^{\text {th }}$ bus. $v_{\min }$ is bus minimum voltage limit. $v_{\max }$ is bus maximum voltage limit

\subsection{Power loss calculation}

The power flow is given by following equations:

$$
\begin{aligned}
& P_{i}=\left|v_{i}\right| \sum_{j=1}^{n}\left|y_{i j}\right|\left|v_{j}\right| \cos \left(\delta_{i}-\delta_{j}-\theta_{i j}\right) \\
& Q_{i}=\left|v_{i}\right| \sum_{j=1}^{n}\left|y_{i j}\right|\left|v_{j}\right| \sin \left(\delta_{i}-\delta_{j}-\theta_{i j}\right)
\end{aligned}
$$

Where $P_{i}$ and $Q_{i}$ are the real and reactive power flowing out of bus i. $\left|y_{i j}\right|$ and $\theta_{i j}$ are the size and angle of admittance. $\delta_{i}$ and $\delta_{j}$ are the angle of voltage at buses $\mathrm{i}$ and $\mathrm{j}$. The $\pi$ model of transmission line is shown in Fig.1.

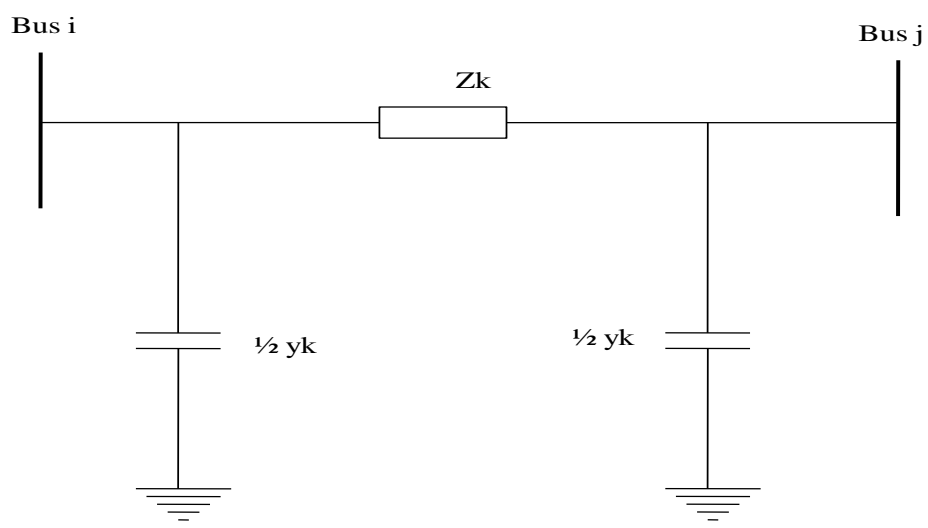

Figure $1 \square$ model of transmission line between buses

The current from $\mathrm{i}^{\text {th }}$ to $\mathrm{j}^{\text {th }}$ bus is given by the relation

$$
I_{i j}=\frac{v_{i}-v_{j}}{z_{k}}-\frac{1}{2} y_{k} v_{i}
$$


Where $\mathbf{z}_{\mathbf{k}}$ and $\mathbf{y}_{\mathbf{k}}$ are the admittance and impedance of line $\mathrm{k}$ and $\mathrm{I}_{\mathrm{ij}}$ is the current from bus $\mathrm{i}$ to bus $\mathrm{j}$.

$$
\begin{gathered}
S_{i j}=v_{i} I_{i j}^{*}, S_{j i}=v_{j} I_{j i}^{*}, \quad S_{\text {lossk }}=S_{i j}-S_{j i}, P_{\text {lossk }}=\operatorname{real}\left(S_{\text {lossk }}\right) \\
P_{\text {lossk }}=\sum_{k=1}^{n} P_{\text {lossk }}
\end{gathered}
$$

\section{Analytical Optimization Methods}

The most useful method of capacitor placement in the power system is the analytical method. This uses the calculus for capacitor placements to calculate the minimum losses and cost savings. This method supposes that the feeder hasn't any sub branches. Its cross-section is the same in all parts and has been distributed equally in the feeder [5]. The analytical methods need a few numerical data from the power system and their application is very easy in practice. The most popular result of analytical methods is the (2/3) rule. According to this rule, in order to come up with the maximum reduction, a capacitor with $(2 / 3)$ drag reactive power from the beginning of the feeder must be installed in a place where its distance is $(2 / 3)$ feeder length in comparison to the beginning of the feeder. Result of the above mentioned method is (2/3) rule. This simplifying hypothesis in the above mentioned method certainly causes errors in obtaining the best results. Sundhararajan et al. [6] shows that how the application of (2/3) rule could be resulted into the increase of losses. Haque et al. [7] proposed an analytical method of minimizing the loss associated with the reactive component of branch currents by placing shunt capacitors. This method first determines a sequence of nodes to be compensated by capacitors. The size of optimal capacitors at the compensated nodes is then determined by optimizing the loss saving equation with respect to the capacitor currents. A voltage independent reactive current model was presented for loss reduction using shunt capacitor by Cook [8]. An analytical method of capacitor placement on primary distribution feeders using reactive current was described by Bae [9]

\section{Fuzzy Logic: A Heuristic Optimization Technique}

Heuristic optimization techniques facilitate solving optimization problems that were impossible or difficult to solve by analytical methods. To solve extremely challenging problems these tools have been combined. Developing solutions with these tools offers two major advantages: (i) The development time is much shorter than when using more traditional approaches and (ii) the systems are very robust. Heuristic optimization Techniques, which promise a near global solution, may be broadly categorized in to three categories, Fuzzy Logic Optimization Technique, Evolutionary Computation Methods and Hybrid Artificial Intelligent Techniques.

The fuzzy logic optimization technique is based on Fuzzy Set Theory (FST) introduced by Zadeh in 1965 dealing with logical reasoning [10]. Fuzzy set theory provides a remedy for any lack of uncertainty in the data. Furthermore fuzzy logic has the advantage of including heuristics and representing engineering judgments into the capacitor allocation optimization process. To apply fuzzy logic to solve the problem, it is necessary to identify the main variables that have influence on the decisions to be taken and quantify their values in relevance levels. A relationship function establishes the profile of these variables by expressing the compatibility degree of each one of them with previously known information. According to these situations, rules are established and necessary actions to a solution are determined. Optimal capacitor planning has been implemented based on the fuzzy algorithm in practical distribution systems [11]. Damodar et al. [12] design a fuzzy approach for identifying the optimal capacitor locations considering two objectives, to minimize the real power loss and to maintain the voltage within the permissible limits. H.Ng et al. [13] proposed the capacitor placement problem by using fuzzy approximate reasoning. Masoum et al. [14] used fuzzy set theory for the discrete optimization problem of fixed shunt capacitor placement and sizing under harmonic conditions.

\section{V.1 Simulated Annealing}

\section{Evolutionary Computation Methods}

Simulated Annealing (SA) is based on analogy of annealing of metal or crystal. SA depends on three important parameters viz. initial temperature $(T)$, cooling rate $(\beta)$ and final temperature $\left(T_{\text {min }}\right)$. The temperature is controlled by the cooling schedule at each iteration. As temperature cools down, the state converges to a solution. The state freely moves to other state at high temperature while it has limitations to move to others at low temperature [15]. SA is a powerful, general-purpose stochastic optimization technique which can theoretically converge asymptotically to the global optimization solution with probability ' 1 '. One main drawback, however, of SA is that it takes much CPU time to find the global optimum.

Ghose et al. [16] investigated the necessity of considering the unbalance and the presence of harmonics in a distribution system while solving the problem with a heuristic SA technique. Application of SA to the capacitor placement problem by means of general purpose heuristic optimization techniques is proposed in [17]. Hsiao et al. [18] applied the SA method to optimal VAR source planning in large-scale power systems. 
W. Jwo et al. [19] presented a hybrid simulated annealing/genetic algorithm (HSAGA) method to reduce the CPU time of SA while retaining its main characteristics, i.e., the ability to get the near-global optimal solution in a finite time. A modified simulated annealing (MSA) technique has been developed for simultaneous improvement of power quality and optimal placement and sizing of fixed capacitor banks in a modern distribution network [20]. The later supplies a mix of linear and nonlinear loads and imposes voltage and current harmonics. These networks include integrated variable-speed wind turbine as a dominant form of distributed generation (DG). The stochastic power output of the wind DG is modeled by Monte-Carlo simulations of the distribution power flow.

\section{V.2 Harmony Search}

Harmony search algorithm (HSA) is a new meta-heuristic algorithm [21]. The Harmony search algorithm is concept from natural musical performance processes. The musicians starting with some harmonies, they attempt to achieve better harmonies by improvisation and create iteratively new good solutions based on past solutions on random modifications. Finally HSA gives optimum value.

The application of the HSA for determining the optimal location and size of shunt capacitors in 9-bus radial distribution systems has been presented in [22]. The test results for the 7 load levels showed that the HS algorithm gives greater power loss reduction and net energy-saving improvement compared to the Genetic algorithm. Sirjani et al. [23] described the application of HSA as a new meta-heuristic optimization technique to determine the optimal location and size of shunt capacitors in a distribution network. The backward/forward sweep power flow is also used to obtain faster power flow solutions.

\section{V.3 Particle Swarm Optimization}

Particle Swarm Optimization (PSO) is a population based and self adaptive meta-heuristic parallel search technique introduced originally by Kennedy and Eberhart in 1995 [24].The PSO technique conducts searches using a population of particles, corresponding to individuals. Each particle represents a candidate solution to the reactive power problem. In a PSO system, particles change their positions by flying a round in a multidimensional search space until a relatively unchanged position has been encountered, or until computational limitations are exceeded. In social science context, a PSO system combines a social- only model and cognition-only model. The social-only component suggests that individuals ignore their own experience and adjust their behavior according to the successful beliefs of the individual in the neighborhood. On the other hand, the cognition-only component treats individuals as isolated beings. A particle changes its position using these models [25].

Tamer Mohamed et al. [26] used a binary particle swarm optimization for discrete optimization problem of optimal capacitor placement in nonlinear loads. Jummat et al. [27] presented an approach for transmission loss reduction by using SVC installation via PSO as the optimization technique. Source code of PSO optimizations technique was developed to determine the optimal sizing of SVC in order to minimize the transmission loss in the system. Besides that, the voltage profiles and cost installation of SVC are considered in the system. Tests are performed on the IEEE 30-bus RTS. Ejal et al. [28] combined a discrete version of PSO with a radial distribution power flow (RDPF) algorithm to form a hybrid PSO (HPSO) algorithm. PSO was employed as a global optimizer to find the global optimal solution, while the RDPF algorithm was used to calculate the objective function and to verify the bus voltage limits. To include the presence of harmonics, the developed HPSO algorithm was integrated with a harmonic power flow (HPF) algorithm. The proposed (HPSOHPF) based approach was tested on an IEEE 13-bus radial distribution system.

\section{V.4 Tabu Search}

The roots of Tabu Search (TS) go back to the 1970's. The TS was first presented in its presentable form by Glover, later it was formalized by him. The basic idea was sketched by Hansen [29]. It is one of the meta-heuristic methods for solving a combinatorial optimization problem [30]-[31]. Meta-heuristics is defined as an optimization algorithm that iteratively uses simple rules or heuristics to evaluate better solutions [32]. TS is based on the hill-climbing method that evaluates the final solution through repeating the process of creating solution candidates in the neighborhood around the initial solution and selecting the best solution among the candidates. The hill-climbing method stops if the solution is not improved. This method can be easily trapped in a local minimum [33]. TS is an extension of the hill-climbing method in a way that it has the adaptive memory called the tabu list, it continues to stay in the tabu list for a while and afterwards a new attribute enters into the tabu list. Each time the iteration count is updated, a new attribute is assigned to the tabu list. In order to obtain optimal solution, TS use intensification and diversification techniques. The frequency counter in the solution structure is used in the intensification and diversification techniques. The frequency counter denotes the times the solution having been visited throughout the solution process. 
Optimal capacitor placement has been achieved in radial feeders using tabu search [34]-[35]. Mori et al. [36] introduced the optimal capacitor placement with hybrid method that utilizes TS. The TS approach has been extended with features from practical heuristic approaches and from other combinatorial approaches, such as genetic algorithms and simulated annealing. Pires et al. [37] described Tabu Search based approach to provide decision support in the problem of capacitor allocation in radial network. The model explicitly considers two conflicting and incommensurate objective functions, related to cost and operations aspects of evaluation. This offers the decision maker (DM) the possibility both to expand the range of potential alternative solutions and to express his/her preferences to select a satisfactory compromise solution.

\section{V.5 Ant Colony Optimization}

The analogy between the way ants look for food and combinatorial optimization problems has given rise to a new computational paradigm, which is called ant colony meta-heuristic. Ants lay down in some quantity an aromatic substance known as pheromone, in their way to food. The pheromone quantity depends on the length of the path and the quality of discovered food source. An ant chooses a specific path in correlation with the intensity of pheromone. The pheromone trail evaporates over time if no more pheromone is laid down. Other ants can observe the pheromone trail and are attracted to follow it. Thus, the path will be marked again and will therefore attract more ants. The pheromone trail on paths leading to rich food source close to the nest will be more frequented and will therefore grow faster. In that way, the best solution has more intensive pheromone and higher probability to be chosen. The described behavior of real ant colonies can be used to solve combinatorial optimization problems by simulation; artificial ants searching the solution space simulate real ants searching their environment. The objective values correspond to the quality of food source. The ant system approach associates pheromone trails to features of the solutions of a combinatorial problem, which can be seen as kind of adaptive memory of the previous solution. Ant system show very good results in each applied area.

Rajeev et al. [38] presented a methodology for employing the ant colony optimization to capacitor selection problem in a balanced three-phase distribution network, in which Newton-Raphson method was used to calculate the cost function. The Ant Colony optimization approach to shunt capacitor placement on distribution systems under switching constraints is presented in [39]. Chang C.F. in [40] presented Ant Colony Optimization based algorithm for the feeder reconfiguration and capacitor placement of distribution systems. The objective of this study was to present new algorithms for solving the optimal capacitor placement problem, the optimal feeder reconfiguration problem and a combination of two.

\section{V.6 Genetic Algorithm}

The theoretical foundations for genetic algorithms (GA) were first described by John Holland [41] and then presented tutorially by David Goldberg [42]. Genetic algorithms are search algorithms based on the process of biological evolution. The genetic algorithm uses Darwin natural selection theorem to find the optimal formula for adapt of the pattern. Generally the genetic algorithm consists of fitness function, selection and change. The most important operators of genetic change, are genetic mutation and cross-over, capable of copying the chromosomes because of their location change and also change of some of the bits of them (Actually they reproduce). At last the chromosome will be generated with maximum fitness amount of the function so the global optimal point is produced in this way [43]. Genetic algorithm or a composition of a genetic method and other ones for optimal designing of the capacitors could be used. Atefeh et al. [44] presented a GA based capacitor placement procedure in ten steps.

M.R. haghifam et al. [45] proposed a GA based method for capacitor allocation in a balanced system which could evaluate uncertainty of loads. In [46] elite based simplex GA hybrid approach combined with multi population GA to determine the location, size and number of capacitors in unbalanced distribution systems is proposed, although the harmonic distorted systems were not considered in this study. The method using index and GA algorithm to determine suitable candidate nodes in distribution systems for capacitor installation has been reported in [47]. A power loss index approach was used to determine the suitability of capacitor placement at each node. The buses with the highest suitability were identified for capacitor placement. Majid Davoodi et al. [48] presented optimal capacitor placement and capacitance computation in the power distribution networks using a method based on GA considering the majority of the influencing factors in its multi-objective target function.

\section{V.7 Plant Growth Simulation Algorithm}

The plant growth simulation algorithm (PGSA) [49] is based on the plant growth process where a plant's trunk grows from its root, some branches will grow from the nodes on the trunk and then some new branches will grow from the nodes on the branches. Such process is repeated, until a plant is formed. Based on an analogy with the plant growth process, an algorithm can be specified where the system to be optimized first 
"grows" beginning at the root of a plant and then "grows" branches continually until the optimal solution is found.

Srinivasa in [50] proposed an efficient approach that employs loss sensitivity factors and PGSA for capacitor placement in the distribution system. The loss sensitivity factors are used to determine the candidate locations of the buses required for compensation. The PGSA is used to estimate the required level of shunt capacitive compensation at the optimal candidate locations to enhance the voltage profile of the system and reduce the active power loss. The proposed algorithm has been tested on 9 and 34 bus system. The Rama Rao et al. [51] proposed a method which deals with initial selection of voltage regulator buses by using Power Loss Indices (PLI) and PGSA for optimal location and number along with tap setting of the voltage regulator, which provides a smooth voltage profile along the network.

\section{V.8 Body Immune Algorithm}

Immune Algorithm is a computational tool which imitates the behavior of human body against the external invasions and is a powerful method in pattern recognition [52]. Immune system is a natural, fast and effective defense mechanism for a host against infection. It includes a complex set of cells and molecules that protect our bodies against infection. Our bodies are under constant attack by antigens that can stimulate the adaptive immune system. Antigens might be foreign, such as surface molecules present on pathogens, or selfantigens, which are composed of cells or molecules of our own bodies [53]. Immune algorithm considers the objective functions and their associated constraints as antigens, which are to be identified by the antibodies, and the solutions which play the rules of antibodies. Affinity factors are defined which indicates the ability of antibodies in recognizing the antigens. Antigen recognition means performance of antibodies in optimizing the objective function while satisfying the constraints. Similar to other evolutionary algorithms, it is an iterative methodology, an initial set of antibodies is generated and then it is tried to improve their response in identifying the antigens. This algorithm has been previously used for solving multi-objective optimization problem in [54].

\section{Hybrid Artificial Intelligent Techniques}

In hybrid intelligent techniques, two or more artificial intelligent techniques are integrated to obtain successful results. A four-stage methodology based on SA, GA, TS and the hybrid GA-Fuzzy logic algorithm to solve a combinatorial optimization problem with a non-differential objective function is presented in [55]. The solution methodologies are preceded by a sensitivity analysis to select the candidate capacitor installation locations.

A combined Fuzzy-GA method for optimal capacitor placement in radial distribution systems and loss minimization is presented in [56]. The proposed method has tested with several systems and considers the loss reduction and voltage profile simultaneously while deciding the location of capacitors. Esmail Limouzade in [57] presented a two-stage methodology of finding the optimal locations and sizes of shunt capacitors for reactive power compensation of radial distribution systems. GA approach is proposed to find the optimal capacitor locations and PSO method is proposed to find the optimal capacitor sizes. A bacterial foraging with a PSO algorithm for optimal capacitor placement considering a multi-objective function is described in [58]. The objective function includes reduction of power losses and installation costs of shunt capacitors and DG units. The solution for different load levels and the utilization of capacitor discrete values were determined for optimization.

\section{Comparison of Various Optimization Techniques}

The PSO is the most popular optimization technique because of its implementation is simple, it has small computational load, and its convergence is fast. PSO is efficient for the solution of difficult problems. However, the PSO algorithm has the disadvantage of premature convergence while solving complex problems. The GA is the second most popular used optimization technique. The GA is considered one of the first metaheuristic techniques for solving optimal capacitor placement. It has drawback such as divergence and local minima problem. Hybrid optimization techniques are used in recent publications. In hybrid methods mostly fuzzy approach is used to find the optimal capacitor location and other techniques such as GA, PSO etc. are used to find optimal capacitor sizes.

\section{Conclusion}

This paper is a survey of work published on different optimization techniques to solve the optimal capacitor placement problem in power transmission and distribution networks to reduce line losses and voltage stability enhancement. The analytical methods are simpler but have the disadvantage of slow computations and poor convergence. The heuristic optimization techniques are fast and good in convergence. These methods are suitable for large and complex networks. The quality of results can be improved by combining the two or more AI techniques called Hybrid AI techniques. This paper provides a general literature survey useful for the research on optimal capacitor placement. 


\section{References}

[1] Ali Elmaouhab, Mohamed Boudour, and Rabah Gueddouche, New evolutionary technique for optimization shunt capacitors in distribution networks, Journal of Electrical Engineering, Vol. 62, No.3,2011, pp.163-167.

[2] (2012) The RVPN website. [Online], Available: http://www.rvpn.co.in/

[3] Capacitor Subcommittee of IEEE Transmission and Distribution Committee, Bibliography on power capacitors 1975_1980, IEEE Trans. Power Apparatus and Systems, Vol. 102, No. 7, July 1983, pp. 2331-2334.

[4] L.Ramesh, S.P.Chowdhury, S. Chowdhury, A.A. Natarajan, and C.T. Gaunt, Minimization of power Loss in distribution networks by different techniques, International Journal of Electrical and Electronics Engineering 3:9, 2009, pp. 521-527.

[5] IEEE VAR Management Working Group of the IEEE System Control Subcommittee, Bibliography on reactive power and voltage control, IEEE Transactions on Power Systems, Vol.2, No.2, May 1997, pp.361-370.

[6] Sundharajan S., and Pahwa A., Optimal selection of capacitors for radial distribution systems using a genetic algorithm, IEEE Transactions on Power Systems, 19(3),1994, pp. 1499-1507.

[7] Haque M. H., Analytical method of minimizing loss associated with the reactive component of branch currents by placing shunt capacitor, IEE Proc. Gener, Trans, Distrib, 146, 1999, pp. 501-505.

[8] R.F. Cook, Calculating loss reduction afforded by shunt capacitor application, IEEE Trans. On Power Apparatus and Systems, PAS-83, 1964, pp. 1227-1230.

[9] Y.G.Bae, Analytical method of capacitor allocation on distribution primary feeders, IEEE Trans. On power Apparatus and Systems, PAS-87(11), 1978, pp. 1232-1238.

[10] L.A. Zadeh, Fuzzy sets, Information and Control, Vol. 8, pp.302-309.

[11] C.T. Su, and C.C. Tsai, A new fuzzy reasoning approach to optimum capacitor allocation for primary distribution systems, in Proceedings of 1996 IEEE on Induatrial Technology Conference, 1996, pp. 237-241.

[12] M.Damodar Reddy, and N.V. Vijaya Kumar,"Optimal capacitor placement for loss reduction in distribution systems using fuzzy and harmony search algorithm," ARPN Journal of Engineering and Applied Sciences, Vol. 7, No. 1, pp.15-19, January 2012.

[13] Ng H.N., Salama M.M.A., and Chikhani A.Y., "Capacitor allocation by approximate reasoning: fuzzy capacitor placement," IEEE Transactions on power Delivery, 15(1), pp. 393-398, 2000

[14] Masoum M., Jafaraian A., Ladjevardi M., Fuchs E., and Grady W., Fuzzy approach for optimal placement and sizing of capacitor banks in the presence of harmonics, IEEE Transactions on Power delivery 19, 2004, pp. 822-829.

[15] A.H.H., Al-Mohammed and I. Elamin, Capacitor placement in distribution systems using artificial intelligent techniques, in IEEE Conf. Power Tech, Italy, 2003, pp. 1-7.

[16] Ghose T., and Goswam S.K., Effects of unbalances and harmonics on optimal capacitor placement in distribution system, Electric Power Systems Research 68, 2, 2003, pp. 167-173.

[17] K.P. Wong, Solving power system optimization problems using simulated annealing, Engineering Applications of Artificial Intelligence Vol. 8, No. 6,Dec. 1995, pp.665-670.

[18] Hsiao YT, Chang HD, Liu CC, and Chen YL., A computer package for optimal multi-objective VAR planning in large scale power systems, IEEE Transactions on Power Systems, 9(2), 1994, pp. 668-676.

[19] Wu-Shun Jwo, Chih-Wen Liu, and Chun-Chang Liu, Large-scale optimal VAR planning by hybrid simulated annealing/genetic algorithm, Electrical Power and Energy Systems 21, 1999, pp. 39-44.

[20] Elmitwally A., Optimal sizing and location of capacitor banks in distorted distribution networks with distributed wind generation, $21^{\text {st }}$ International Conference on Electricity Distribution, 2011, pp. 1-4.

[21] R. Srinivasa Rao, A hybrid approach for loss reduction in distribution systems using harmony search algorithm, International Journal of Electrical and Electronics Engineering,2010, pp. 4-7.

[22] R. Sirjani, A.Mohamed, and H. Shareef, An improved harmony search algorithm for optimal capacitor placement in radial distribution systems, in IEEE Conf. Power Engineering and Optimization, Malaysia, 2011, pp. 323-328.

[23] R. Sirjani, A.Mohamed,and H. Shareef, Optimal capacitor placement in radial distribution system using harmony search algorithm, Journal of Applied Sciences 10, No.23,2010, pp. 2996-3006.

[24] J.Kennedy, and R.C. Eberhart, Particle swarm optimization, in IEEE Conf. Neural Networks, Australia, 1995, pp.502-505.

[25] Abido M.A., Optimal design of power-system stabilizers using particle swarm optimization, Energy Conversion, IEEE Transactions, Vol. 17 Issue.3,Sep.2002, pp. 406-413.

[26] Tamer Mohamed Khalil, Hosam K.M. Youssef, and M.M. Abdel Aziz, Optimal capacitor placement on radial distribution feeders in presence of nonlinear loads using binary particle swarm optimization, CIRED $19^{\text {th }}$ International Conference on Electricity distribution, Vienna, 21-24 may 2007, Paper No. 180.

[27] S.A. Jummat, Ismail Musirin, Muhammad Murtadha Othaman, and Hazlie Mokhlis, PSO Based technique for loss minimization considering voltage profile and cost function, The $5^{\text {th }}$ International Power Engineering and Optimization Conference, Selangor, Malaysia, 6-7 June-2011, 978-1-4577-0353-9/11 @2011 IEEE.

[28] Ejal A., and Elhawary M.E., Optimal capacitor placement and sizing in unbalanced distribution system with harmonics consideration using particle swarm optimization, IEEE Transactions on Power Delivery 25, No. 3,2010, pp. 1734-1741.

[29] Tilak Thakur, and Jaswanti Dhiman, A tabu search algorithm for multi-objective purpose of feeder reconfiguration, Journal of Electrical and Electronics Engineering Research (2141-2367), Vol 3(4), June 2011, pp. 71-79.

[30] B. Dengiz, and C. Alabas, Simulation optimization using tabu search, in IEEE Conf. Winter Simulation, 2000, pp.805-810.

[31] Glover F., Tabu search Part II, ORSA Journal on Computing 2, No. 1,1990, pp. 4-32.

[32] Osman H.I., and Kelly J.P., Meta-heuristics: theory \& applications, (Kluwer Academic Publishers, Netherlands, 1996).

[33] Gallego R.A., Monticelli A.J., and Romero R., Optimal capacitor placement in radial distribution networks, IEEE transactions on Power Systems 16, No.4,2001, pp.630-637.

[34] Y.C. Huang, Solving the capacitor placement problem in a radial distribution system using tabu search approach, IEEE Trans On Power Delivery, 11(4), 1996, pp.1868-1873.

[35] H.Mori, and Y.Ogita, Parallel tabu search for capacitor placement in radial distribution systems, Proc. of IEEE Power Engineering Society Winter Meeting, 2000, 4, pp. 2334-2339.

[36] Mori H., and Tsunokawa S., Variable neighborhood tabu search for capacitor placement in distribution systems, IEEE International Symposium on Circuits and Systems, ISCAS- 2005, pp. 4747-4750.

[37] Dulce F. Pires, C. Henggeler Antunes, and A. Gomes Martins, A tabu search multi-objective approach to capacitor allocation in radial feeder, $4^{\text {th }}$ Metaheuristics International Conference, July 16-20, 2001, Porto, Portugal.

[38] Rajeev Annaluru, Sanjoy Das, and Anil Pahwa, Multi-level ant colony algoritm for optimal placement of capacitors in distribution systems, IEEE Transaction 0-7803-8515-2/04, 2004. 
[39] S. Bouri, A.Zeblah, A. Ghoraf, S. Hadjeril, and H. Hamdaoui, Ant colony optimization to shunt capacitor allocation in radial distribution systems, Acta Electrotechnica et Informatica, Vol. 5, no.4,2005, pp. 1-9.

[40] Chang C.F., Reconfiguration and capacitor placement for loss reduction of distribution systems by ant colony search algorithm, IEEE Transactions on Power Systems 23, No.4, 2008, pp. 1747-1755.

[41] D.E. Goldberg, Ggenetic Algorithm in Search Optimization and Machine Learning, (Massachusetts, Addison -Wesley, 1989).

[42] Turan Gonen, Electric Power Distribution System Engineering, (McGraw-Hill, 1986).

[43] Delfanti M., Granelli G.P., Marannino P., and Montagna M., Optimal capacitor placement using deterministic and genetic algorithms, IEEE Transactions on Power Systems, Vol. 15, No. 3, August 2000, pp. 1041-1046.

[44] Atefeh Poushafie, Mohsen. Saniei, S.S. Mortazavi, and A. saeedian, Optimal compensation of reactive power in the restructured distribution network, World Academy of science, Engineering and Technology 30, 2009, pp. 119-122.

[45] M.R. haghifam, and O.P. Malik, Genetic algorithm based approach for fixed and switchable capacitors placement in distribution systems with uncertainty and time varying loads, IET Generation, Transmission and Distribution, Vol. 1, No.2, 2007, pp. $244-252$.

[46] K.H. Kim, S.B. Rhee, S.N. Kim, and S.K. You, Application of ESGA hybrid approach for voltage profile improvement by capacitor placement, IEEE Transactions on Power delivery, Vol. 18, No. 4, 2003, pp. 1516-1522.

[47] Swarnkar A., Gupta N., and Niazi K.R., Optimal placement of fixed and switched shunt capacitors for large-scale distribution systems using genetic algorithms, Innovative Smart Grid Technologies Conference, 2010, pp. 1-8.

[48] Majid Davoodi, Mohsen Davoudi, Iraj Ganjkhany, and Ali Aref, Optimal capacitor placement in distribution networks using genetic algorithm, Indian Journal of Science and Technology, Vol. 5, No. 7,July 2012, pp.3054-3058.

[49] Chu Wang, H.Z.Chengand and L.Z. Yao, Optimization of network reconfiguration in large distribution systems using plant growth simulation algorithm, DRPT 2008 Conference, Nanjing, China, 6-9 April 2008, pp. 771-774.

[50] R. Srinivasa Rao, Optimal capacitor allocation for loss reduction in distribution system using fuzzy and plant growth simulation algorithm, International Journal of Electrical and Computer Engineering 5:2, 2010, pp.71-77.

[51] P.V.V. RamaRao, S.Sivanagaraju, and P.V. Prasad, Voltage regulator placement in radial distribution network using plant growth simulation algorithm, $16^{\text {th }}$ National Power System Conference, 15-17 December, 2010, Hyderabad India, pp.284-288.

[52] Engelbrecht AP. Computational intelligence: an introduction, (John Willey \& Sons, University of Pretoria South Africa, 2007).

[53] Y. Tsujimura, and M.Gen, Entropy-based genetic algorithm for solving TSP, in 1998 Second International Conference on Knowledge-Based Intelligent Electronic Systems, No. 2, 21-23, April, 1998, pp. 285-290.

[54] Tan K,Goh C., Mamum A., and Eu E., An evolutionary artificial immune system for multi-objective optimization, European Journal of Operational Research, 187(2), 2008, PP.371-392.

[55] Ladjavardi M.,and Masoum M.A.S., Genetically optimized fuzzy placement and sizing of capacitor banks in distorted distribution networks, IEEE Transactions on Power delivery 23, No.1,2008, 449-456.

[56] P.V. Prasad, S.Sivanagaraju, and N.Sreenivasulu, A fuzzy-genetic algorithm for optimal capacitor placement in radial distribution systems, ARPN Journal of Engineering and Applied Sciences, Vol. 2 No.3, June 2007, pp.28-32.

[57] Esmail Limouzade, Optimal placement of capacitors in radial distributiom system using a GA-PSO method, Trends in Advanced Science and Engineering, TASE-5(1),2012,pp. 60-65.

[58] Mohkami H., Hooshmand R., and Khodabakhshian A., Fuzzy optimal placement of capacitors in the presence of non-linear loads in unbalanced distribution networks using BF-PSO algorithm, Applied Soft Computing 11, 2011, pp. 3634-3642. 